\title{
Improvements on colony morphology identification towards bacterial profiling
}

\author{
Ana Margarida Sousa, Idalina Machado, Ana Nicolau, Maria Olívia Pereira* \\ Institute for Biotechnology and Bioengineering (IBB), Centre of Biological Engineering, University of Minho, Campus de Gualtar, 4710-057 Braga, Portugal
}

\section{A R T I C L E I N F O}

\section{Article history:}

Received 8 July 2013

Received in revised form 26 September 2013

Accepted 30 September 2013

Available online 9 October 2013

\section{Keywords:}

Colony morphology

Bacterial profiling

Pseudomonas aeruginosa

Biofilms

Small colony variants

\begin{abstract}
A B S T R A C T
Colony morphology may be an indicator of phenotypic variation, this being an important adaptive process adopted by bacteria to overcome environmental stressors. Furthermore, alterations in colony traits may reflect increased virulence and antimicrobial resistance. Despite the potential relevance of using colony morphological traits, the influence of experimental conditions on colony morphogenesis has been scarcely studied in detail. This study aims to clearly and systematically demonstrate the impact of some variables, such as colony growth time, plate colony density, culture medium, planktonic or biofilm mode of growth and strain genetic background, on bacterial colony morphology features using two Pseudomonas aeruginosa strains. Results, based on 5-replicate experiments, demonstrated that all variables influenced colony morphogenesis and 18 different morphotypes were identified, showing different sizes, forms, colours, textures and margins. Colony growth time and composition of the medium were the variables that caused the highest impact on colony differentiation both derived from planktonic and biofilm cultures. Colony morphology characterization before $45 \mathrm{~h}$ of incubation was considered inadequate and TSA, a non-selective medium, provided more colony diversity in contrast to $P$. aeruginosa selective media. In conclusion, data obtained emphasized the need to perform comparisons between colony morphologies in equivalent experimental conditions to avoid misinterpretation of microbial diagnostics and biomedical studies. Since colony morphotyping showed to be a reliable method to evaluate phenotypic switching and also to infer about bacterial diversity in biofilms, these unambiguous comparisons between morphotypes may offer a quite valuable input to clinical diagnosis, aiding the decision-making towards the selection of the most suitable antibiotic and supportive treatments.
\end{abstract}

(c) 2013 Elsevier B.V. All rights reserved.

\section{Introduction}

Isolation of specimens by bacteriological techniques such as the culturing in selective or differential media is a routine procedure, especially in clinical laboratories. Growing on agar surfaces, microorganisms form colonies whose appearance helps the clinicians and researchers to identify genera or even species. One of the most intriguing aspects of this approach is the observation of similar colony patterns in different systems and the existence of distinct patterns when culturing a sole strain in analogous conditions. The large number of reckonable patterns turns the identification of colony morphologies a real challenge for microbiologists, clinicians and technicians.

Rapid technological advances in bacterial identification methods have occurred providing a formidable wide range of techniques to detect, identify and differentiate bacteria. Molecular methods such as ELISA, PCR and MALDI-TOF MS have introduced great improvements in bacterial identification as they contributed to speed up the analysis

\footnotetext{
* Corresponding author at: Institute for Biotechnology and Bioengineering (IBB), Centre of Biological Engineering, University of Minho, 4710-057 Braga, Portugal. Tel.: + 351253 604402; fax: + 351253678986 .

E-mail address: mopereira@deb.uminho.pt (M.O. Pereira).
}

and the reduction of handling (Weile and Knabbe, 2009). Despite the technological advances, culture-based strategies are still necessary to obtain information about the microbiological effect of the antibiotic courses, by determining the number of bacteria before and after antibiotic administration, as well as to determine whether phenotypic selection is occurring. Antibiotic treatments may exert a pressure selection over bacteria that may not be detected using molecular methods. Atypical colony morphologies can often exhibit unusual biochemical and metabolic features turning clinical identification into a challenge. For instance, Staphylococcus aureus small colony variants (SCV) have altered metabolic activity, thus interfering with the results of biochemical tests as the negative results of coagulase tests (Hilmi et al., 2013). Therefore, colony morphology characterization often complements conventional microbial identification detecting intra-strain diversity (Qamer et al., 2003).

Intra-population diversity generated by bacterial phenotypic and genetic adaptation may be beneficial since it allows both evolution and adaptation to new and changing environments increasing the chances of bacteria survival (Boles et al., 2004; Goerke et al., 2007; Yachi and Loreau, 1999). Alterations in colony morphological traits can be a macroscopic manifestation of the several biological strategies adopted by microorganisms to face stress conditions, as starvation, 
depletion of oxygen, antibiotics and host defences (Sousa et al., 2011). Furthermore, the different aspect of colonies may reflect differences in virulence (Davies et al., 2007; Martin et al., 1993; Rossignol et al., 2009; Tannaes et al., 2000), antimicrobial resistance (Lewis, 2005; Massey et al., 2001; Sousa et al., 2011) and persistence (Balaban et al., 2004; Singh et al., 2009; Spoering and Lewis, 2001). Therefore, and despite being described by several authors as oldfashioned (Braga et al., 2013; Weile and Knabbe, 2009), colony morphology characterization can provide valuable insights into individual microbial diversity, both derived from genetic changes or reversible changes (Sousa et al., 2011).

In chronic infections, including cystic fibrosis (CF), the existence of multiple colony morphology variants is recurrent. One of the most important clinical features in $\mathrm{CF}$ is the Pseudomonas aeruginosa conversion from non- to mucoid form, being the later phenotype more difficult to eradicate (Hogardt and Heesemann, 2010; Lyczak et al., 2002). Mucoid variants are markedly more resistant to antibiotics, such as gentamicin, aminoglycosides, ciprofloxacin and imipenem, or can be even multiresistant (Agarwal et al., 2005; Manno et al., 2005). Several other morphotypes have been identified in bacteria related to chronic and acute infections. The most common and best studied are SCV (Haussler, 2004; Haussler et al., 1999, 2003; Hoffman et al., 2006; Massey et al., 2001; Proctor et al., 2006; Wellinghausen et al., 2009), the rough (small) colonies (Drenkard and Ausubel, 2002) and the hyperpiliated colonies (Deziel et al., 2001; Haussler et al., 2003).

Biofilm formation, the microbial organization in multicellular communities, is another relevant strategy used by microorganisms to face stress conditions and to introduce microbial diversity (Costerton et al., 1987; Donlan, 2002; Drenkard, 2003; Lewis, 2001; Stewart, 2002). Bacteria may develop some phenotypic changes to facilitate the growth as a biofilm (Sauer et al., 2002), being these changes observed when bacteria are recovered from biofilms, plated on agar media and form colonies with distinct morphological patterns (Sousa et al., 2011).

Apart from obvious morphological differences that have been reported, the conditions in which evaluation has been performed were different among published works. This variety is especially significant in what the medium used and the growth time for colony development is concerned. To obtain new insights on colony morphology identification, this study used two $P$. aeruginosa strains (a reference strain and a clinical isolate) to perform a detailed and broad evaluation of colony morphologies during their development experiencing different growth times, plate densities, culture media and mode of growth, including planktonic and biofilm lifestyle. This study aimed at determining the impact of the experimental conditions on each colony morphological features, including form, margin, texture, size and colour, in a systematic way.

\section{Methods}

\subsection{Bacterial strains and culture conditions}

P. aeruginosa ATCC 10145 and a clinical isolated from medical equipment (from now on referred as clinical strain) were used throughout this study. Bacteria were routinely cultured on tryptic soy broth (TSB) or agar (TSA) medium at $37^{\circ} \mathrm{C}$. All strains were preserved in cryovials (Nalgene) at $-80 \pm 2{ }^{\circ} \mathrm{C}$. Prior to each experiment, bacterial cells were grown on TSA plates for $24 \mathrm{~h}$ at $37^{\circ} \mathrm{C}$. The use of a reference strain and a clinical isolate ensured the different genetic background.

\subsection{Planktonic cultures}

Planktonic bacteria grew overnight in TSB at $37^{\circ} \mathrm{C}$ and $120 \mathrm{rpm}$. Cell suspension of each strain was washed twice in PBS by centrifugation (9000 g, $5 \mathrm{~min}$ ) and further serially diluted and plated on solid media.

\subsection{Biofilm formation}

Biofilms were developed as previously described (Stepanovic et al., 2000). Briefly, bacteria were grown overnight on TSB at $37^{\circ} \mathrm{C}$. Cell suspension of each strain was diluted in TSB to obtain $10^{7} \mathrm{CFU} / \mathrm{mL}$ as final concentration. Afterwards, the bacterial suspension was transferred to a 96-well polystyrene microtiter plate where biofilms were developed aerobically on a horizontal shaker $(120 \mathrm{rpm})$ at $37{ }^{\circ} \mathrm{C}$ for $24 \mathrm{~h}$. After that, biofilms were sonicated into sterile water and vortexed to homogenize. Finally biofilm-cells were serially diluted with PBS and spread plated on solid media.

\subsection{Observation and classification of colony morphology}

To assess the impact of the solid media composition on colony morphology features, bacteria were serially diluted and plated on different solid media in air conditions, including TSA (15 g/L, Liofilchem), Pseudomonas isolation agar (PIA, $45 \mathrm{~g} / \mathrm{L}$, Fluka) and cetrimide (CET, $45.5 \mathrm{~g} / \mathrm{L}$, Merck) plus $10 \mathrm{~mL} / \mathrm{L}$ of glycerol, at $37^{\circ} \mathrm{C}$. It was observed that the amount of solid medium in the plate had impact on colony morphogenesis. Therefore, the height of solid media per plate was standardized to values nearby $0.5 \mathrm{~cm}$ (approximately $15 \mathrm{~mL}$ per $90 \mathrm{~cm}$ plate). To evaluate the influence of the origin of the bacteria on its colony morphology, bacteria coming from biofilms and from planktonic cultures were used. Plates with different colony numbers were observed to infer about the role of colony density on morphology differentiation. Finally, different times of incubation were used to assess the effect of growth time: after $15,24,30,45$ and $50 \mathrm{~h}$ of incubation. Colonies were observed by directly placing the Petri plates under a magnifying glass (Olympus SZCTV) and recorded with a CCD camera (AVC, D5CE; Sony, Tokyo, Japan). The identification and classification of colony morphotypes were carried out using five parameters: colony size, form, colour, texture and margin, according to Table 1 . A phenotypic variant was considered when it differed in at least one of the referred morphological parameters. All experiments were performed 5 times.

\section{Results}

To describe each colony regarding the distinct morphological characteristics, the following terms were used as synonymous: colony morphology, colony type, colony variant and morphotype, all meaning a group of bacteria grown from a single cell on agar surface, exhibiting a typical colonial pattern. It must be remarked for further studies that this definition does not exclude the possibility that different strains or species exhibit the same morphotype or that a strain or species exhibits more than one morphotype. All morphotypes presented were observed at least in 3 of the 5 replicates.

Table 1

Morphological features used to characterize $P$. aeruginosa colony morphologies.

\begin{tabular}{ll}
\hline Class & Sub-class \\
\hline Colony Form & Circular \\
Colony Margin & Irregular \\
& Entire \\
Colony Texture & Irregular \\
& Smooth \\
Colony Size & Rough \\
& Wrinkled \\
Colony Colour & Small \\
& Large \\
& White \\
& Brown \\
& Yellow \\
\hline
\end{tabular}

\footnotetext{
a Colonies were considered small if presented diameter is
} below $3 \mathrm{~mm}$ and large if presented diameter is above $3 \mathrm{~mm}$. 


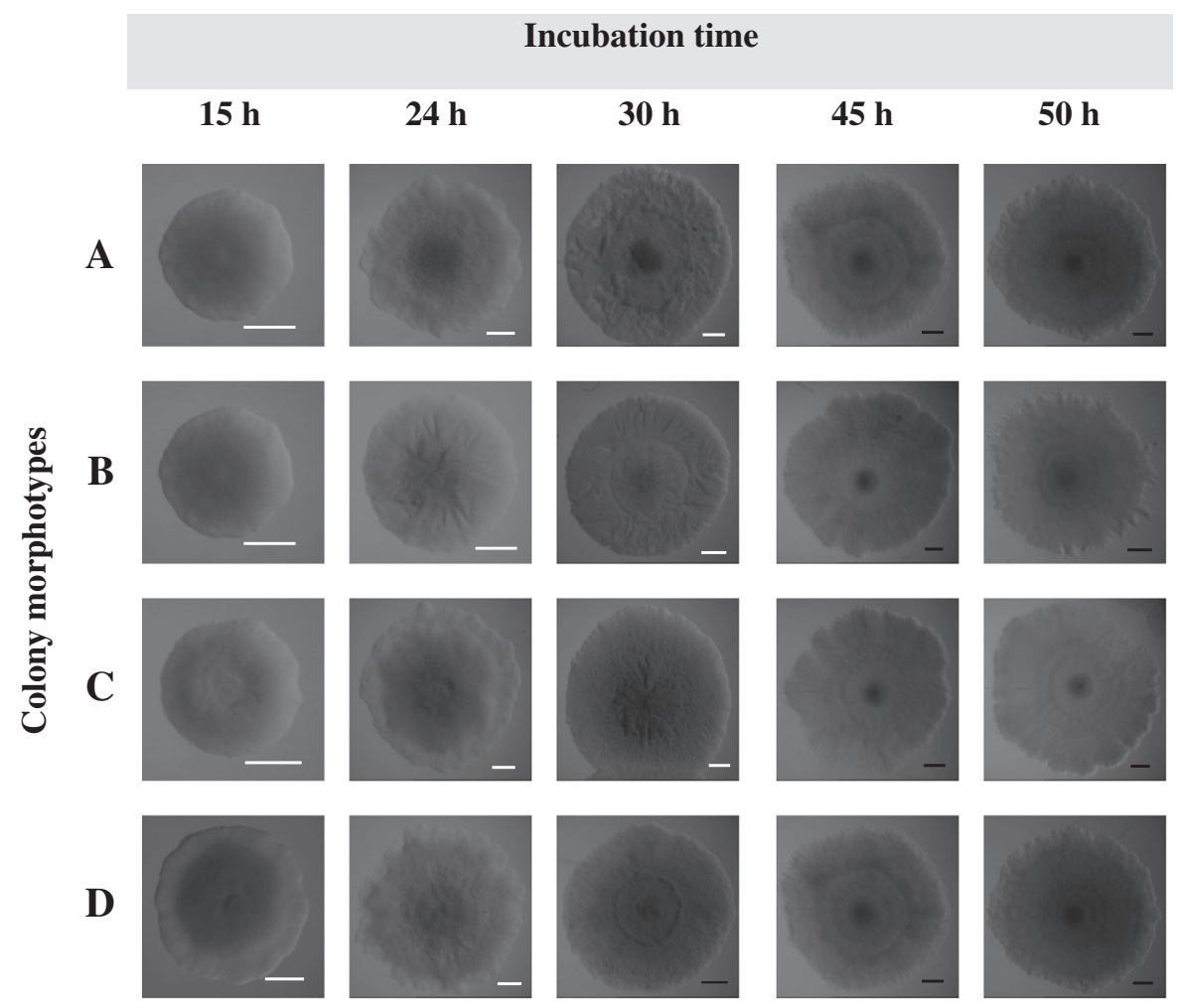

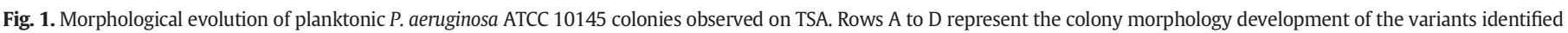

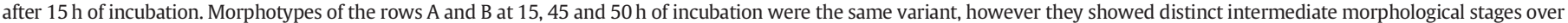
time (at 24 and $30 \mathrm{~h}$ ). All morphotypes were observed at least 3 times of the 5 performed. White bars $=0.5 \mathrm{~mm}$; black bars $=1 \mathrm{~mm}$.

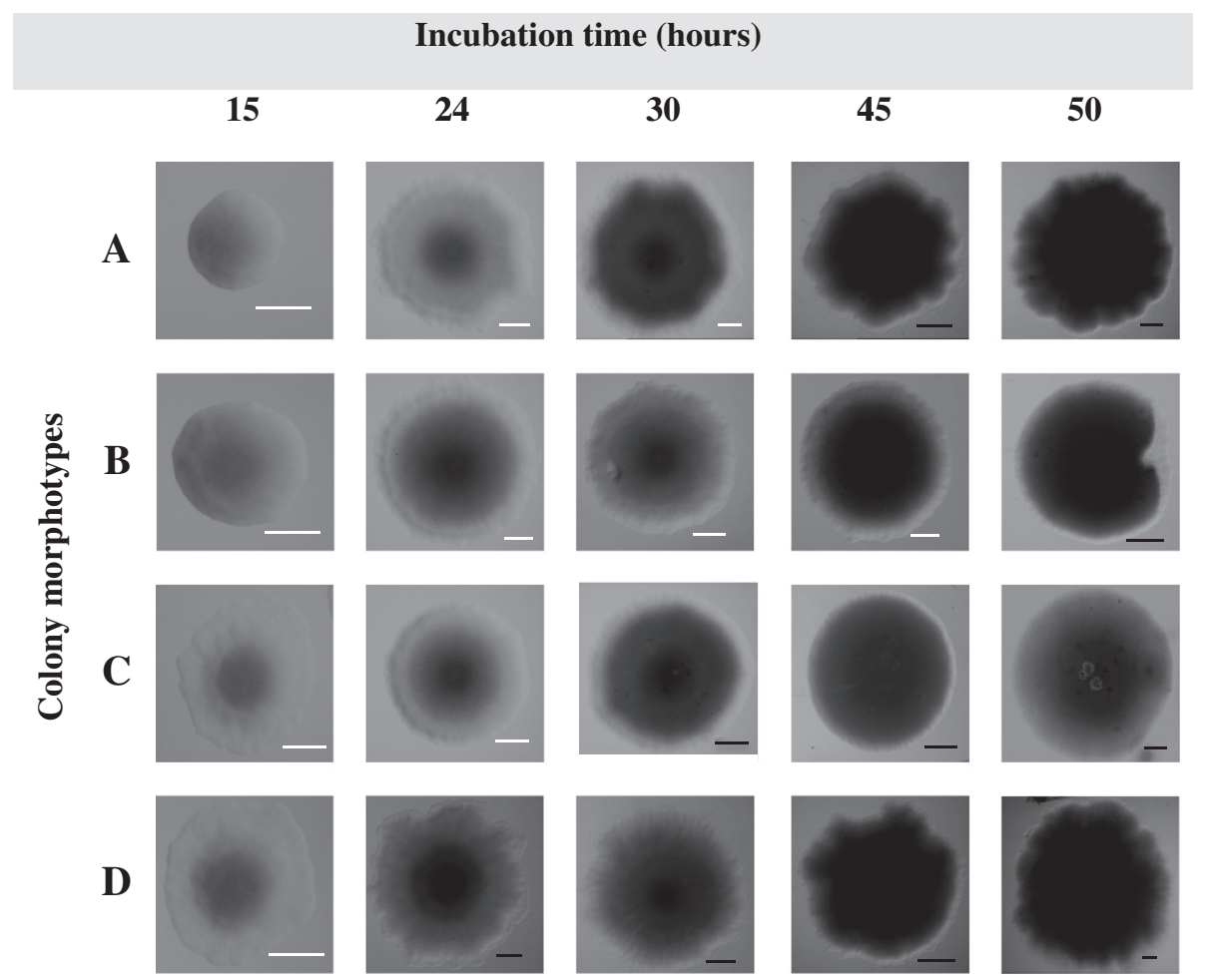

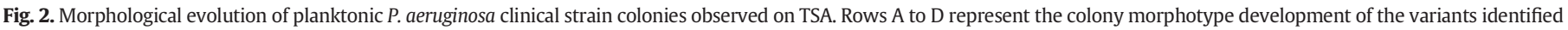

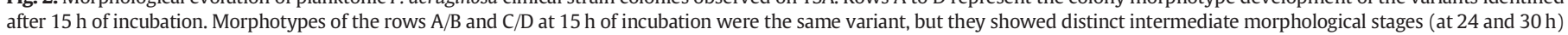
ending into different variants, $\mathrm{B}=\mathrm{C}$ and $\mathrm{A}=\mathrm{D}$. All morphotypes were observed at least 3 times of the 5 performed. White bars $=0.5 \mathrm{~mm}$; black bars $=1 \mathrm{~mm}$. 


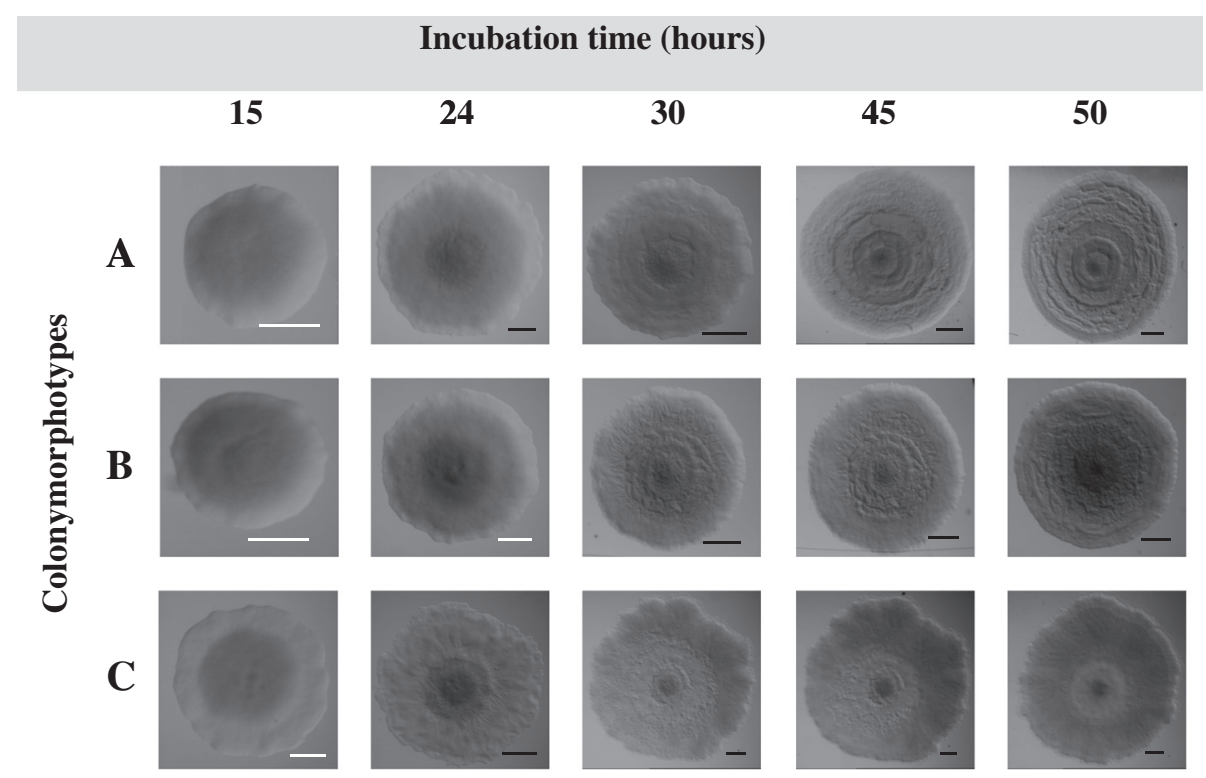

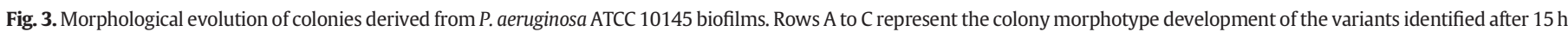

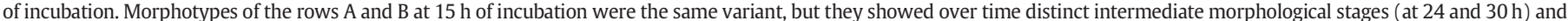
ended as different variants (columns 45 and $50 \mathrm{~h}$ ). All morphotypes were observed at least 3 times of the 5 performed. White bars $=0.5 \mathrm{~mm}$; black bars $=1 \mathrm{~mm}$.

\subsection{Effects of growth time on colony morphogenesis}

Planktonic and biofilm bacteria were cultured on solid media plates and colonies were examined after $15,24,30,45$ and $50 \mathrm{~h}$ of incubation at $37^{\circ} \mathrm{C}$. These intervals of time allowed the observation of the sequential stages of colony morphological features during growth on the different solid media (data shown just addressing TSA plates, Figs. 1, 2, 3 and 4). After $15 \mathrm{~h}$ of incubation, heterogeneity among colony morphologies was only incipient. Between 15 and $45 \mathrm{~h}$ of incubation, colonies changed their appearance, the planktonic $P$. aeruginosa ATCC exhibiting identical final morphotypes (Fig. 1) while the clinical strain revealed completely distinct biofilm-variants (Fig. 4A-H). An interesting result was that, independent of the strain or the type of morphotype, after $45 \mathrm{~h}$, no morphological changes were observed, apart from the increase of the colony size, meaning that at $45 \mathrm{~h}$ the colony morphogenesis was complete and all morphological traits were well defined. This evidence was verified in all solid media tested (TSA, PIA and CET). Therefore, further analyses of colony morphology were performed considering the traits exhibited at $45 \mathrm{~h}$ of growth.

\subsection{Effects of plate colony density on colony morphogenesis}

During colony evaluation, it was observed that colonies growing close to each other altered their development (Fig. 5). A colony density higher than 20 colonies per agar plate resulted in close neighbouring growth and thus in limited and structured colony formation when compared with more distant or isolated colonies. For instance, distant colonies of planktonic $P$. aeruginosa clinical strain that exhibited circular form, undulate margin, rough texture and large size (Fig. 5C), when grown in plates with 20 to 100 colonies exhibited circular form, irregular margin, rough texture and small size (Fig. 5B) and in plates with more than 100 colonies exhibited undistinguished form and margin, rough texture and undetermined size (Fig. 5A). To ensure unbiased data, morphology characterization was performed in plates with a maximum of 15 colonies.

\subsection{Effects of culture medium composition on colony morphogenesis}

Planktonic and biofilm-associated P. aeruginosa were plated onto different solid media (TSA, PIA and CET) in order to assess the role of nutritional composition on colony morphology definition. The results showed that solid media clearly influenced colony morphogenesis. Both $P$. aeruginosa strains, either from planktonic and biofilm cultures, when plated on TSA, PIA and CET exhibited distinct colony morphologies according to the media used (Table 2). It was verified that the features margin, texture and colour were the most affected. The most relevant result is the high diversity of morphotypes obtained from the biofilm cultures of both strains, namely the clinical strain that originated 5 fold more colony morphotypes when plated onto TSA than the reference strain.

\subsection{Impact of the mode of growth on colony morphogenesis and diversity}

Based on the fact that biofilm formation is usually originated by planktonic cells, it was considered of utmost importance to identify the colony morphotypes of the initial population that originated the biofilms.

As TSA was the medium where higher colony diversity was observed, thus the data analysis was just focused on morphotypes observed on TSA. An increased number of different colony morphologies was observed in biofilm-associated bacteria compared to planktonic counterparts. ATCC biofilms encompassed three distinct large and circular colony morphologies: a wrinkled and concentric variant with an entire margin (Fig. 3A, column $45 \mathrm{~h}$ ); a rough colony variant with irregular margin (Fig. 3B, column $45 \mathrm{~h}$ ); and the third variant (Fig. 3C, column $45 \mathrm{~h}$ ) similar to the planktonic counterpart (Fig. 1, column $45 \mathrm{~h}$ ). In

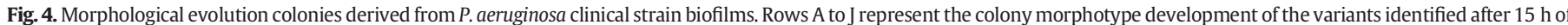

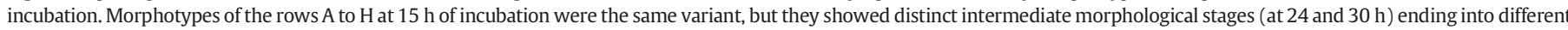

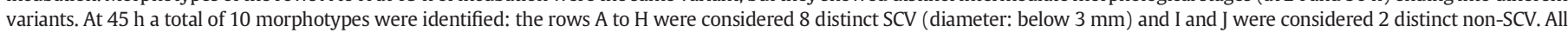
morphotypes were observed at least 3 times of the 5 performed. White bars $=0.5 \mathrm{~mm}$; black bars $=1 \mathrm{~mm}$. 


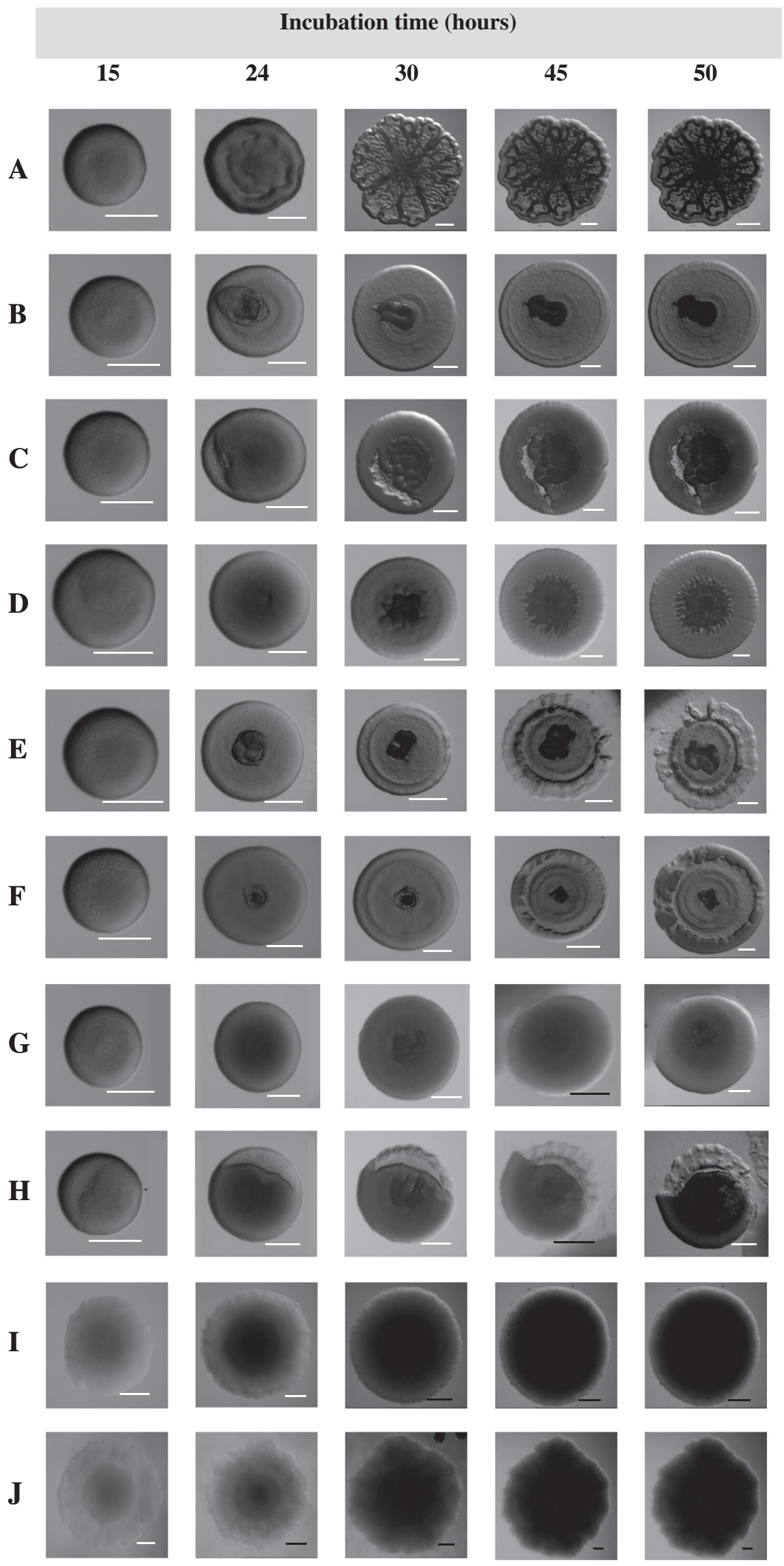


A

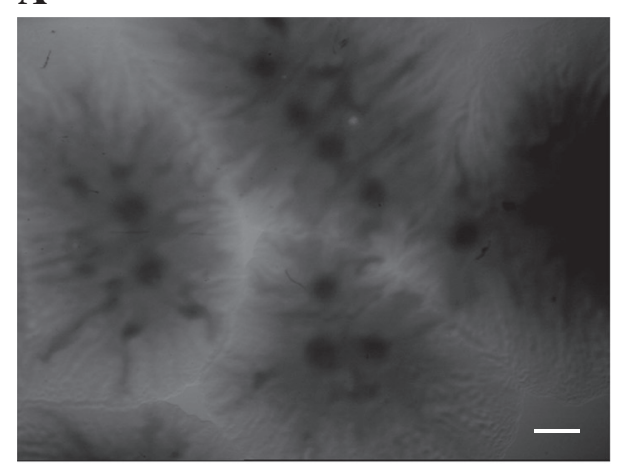

B

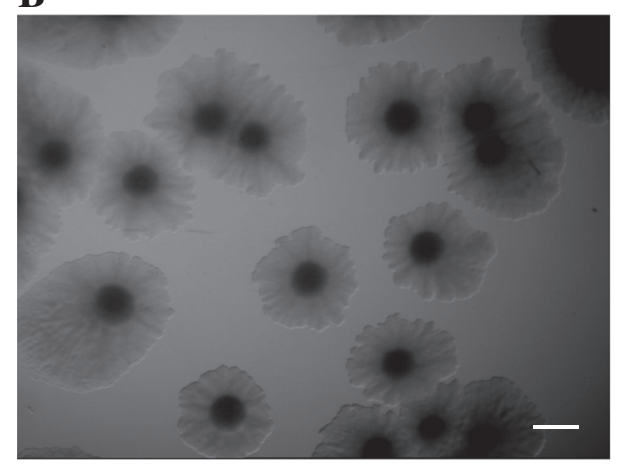

C

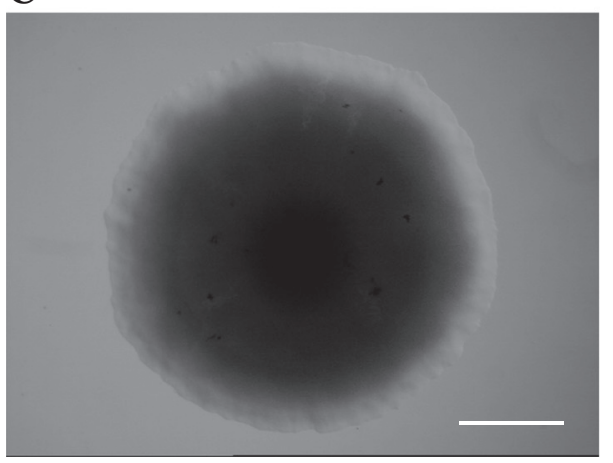

Fig. 5. Colony morphologies of planktonic $P$. aeruginosa clinical strain after $30 \mathrm{~h}$ of incubation on plates with: (A) more than 100 colonies; colonies exhibited undistinguished form and margin due to high colonies density on the plate, rough texture and undetermined size; (B) approximately 30 colonies; colonies exhibited circular form, irregular margin, rough texture and small size; (C) 20 or less colonies; colonies exhibited circular form, undulate margin, rough texture and large size, distinct morphology in contrast to (a) and (b). These experiments were performed 5 times. White bars $=1 \mathrm{~mm}$.

contrast, the planktonic reference strain gave rise to a homogeneous population composed of large and circular colonies, with a small marked centre, irregular margin and with a sheath (Fig. 1, column $45 \mathrm{~h}$ ). The surface showed to be predominantly rough with some small wrinkled zones. These colonies were yellow when plated on TSA.

Much higher diversity was detected from the biofilm-associated bacteria developed by the clinical strain. Eight distinct SCV, colonies with less than $3 \mathrm{~mm}$ of diameter (Fig. $4 \mathrm{~A}-\mathrm{H}$, column $45 \mathrm{~h}$ ), and two larger colony variants were observed (Fig. $4 \mathrm{I}$ and J, column $45 \mathrm{~h}$ ). In turn, the planktonic clinical strain originated a heterogeneous population just composed by two large yellowish colony variants: smooth with entire margins (Fig. 2B and C, column $45 \mathrm{~h}$ ), and by smooth variants with irregular margins (Fig. $2 \mathrm{~A}$ and D, column $45 \mathrm{~h}$ ). The observation of new colony variants in the case of biofilm-associated bacteria, suggests that a fraction of the planktonic cells changed phenotypically when growing as a biofilm.

\subsection{Effect of genetic background on colony morphogenesis}

Despite belonging to the same species, the genetic background exerted significant influence on the definition of colony traits as shown in Table 2. Colonies of both strains in the same experimental conditions shared some characteristics and differed in others. For instance, planktonic cultures resulted mostly in colonies that shared the large dimension and the circular form, but were clearly different in other traits, such as the texture and the margin. Another interesting result was the different ability of the two strains to generate colony variants: the clinical strain originating 5 fold more colony morphotypes-biofilm associated when plated onto TSA than the reference strain.

\section{Discussion}

The recognition of typical colony morphologies is crucial, among others, for clinical diagnosis. Scientific and clinical laboratories frequently use the colony morphology displayed by bacteria on agar media as an auxiliary means to identify bacterial species because of their different and specific growth patterns.

Changes in colony morphology are gaining attention because they are thought to be the expression of the adaptation to different environments, thus hampering the pathogen identification based on morphological traits. Distinct colony morphotypes may arise and their erroneous identification and/or characterization may significantly influence the clinical diagnosis. On the other hand, alterations in colony morphology traits may reveal cellular alterations caused by phenotypic switching that confer ensured virulence, antimicrobial resistance and persistence (Massey et al., 2001; Sousa et al., 2011). Phenotypic switching refers to a reversible switch between two phenotypic states analogous to an ON/OFF mechanism, i.e., microorganisms can interchange between states. The advantage of phenotypic switching is the generation of heterogeneous and dynamic populations that can overcome stressful challenges without the fitness costs of irreversible mutations. This process has been studied based on colony morphology evaluation (Be'er et al., 2011; Chantratita et al., 2007; Massey et al., 2001).

The switch of mode of growth from planktonic to biofilm implies as well cellular alterations that can be observed in colony morphology variation. Biofilms have been increasingly recognized as an important issue in human disease due to their notorious resistance, achieving 10 to 1000 fold higher tolerance to antimicrobial agents than the corresponding planktonic bacteria (Davies, 2003). Biofilms encompass a wide range of microniches with specific biological activities that may somewhat translate the well-known biofilm heterogeneity. Stewart and Franklin (2008) reported that in a mature biofilm at least three distinct physiological states can be anticipated: cells near the biofilm-bulk-fluid or in the more superficial layer, presenting similarities with planktonic cells; cells in the middle zone; and cells in the deeper zone. In fact, the present results reinforce the potential of colony morphology characterization to discriminate the biofilm population diversity since several colony morphotypes were isolated from biofilms (Figs. 3 and 4).

The actual interpretation of the function of colony morphotypes isolated from clinical samples or identified in vitro experiments is mainly based on results in comparison among reports. A detailed analysis of methodological procedures of these reports revealed that experimental conditions are highly variable among reports. For instance, $P$. aeruginosa colonies have been characterized after being grown on different agar media, and also with different medium supplements, as well as after different growth times (Hay et al., 2009; Rakhimova et al., 2008; Starkey et al., 2009). This has also happened with other species as S. aureus (Norstrom et al., 2007; Schneider et al., 2008), Streptococcus pneumoniae (Allegrucci and Sauer, 2007; Weiser et al., 1996) and Enterococcus 
Table 2

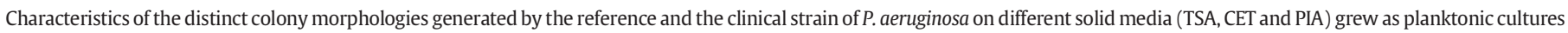
and biofilms.

\begin{tabular}{|c|c|c|c|c|c|c|}
\hline & Media & Form & Margin & Texture $^{\mathrm{a}}$ & Size $^{b}$ & Colour \\
\hline \multicolumn{7}{|l|}{ Planktonic } \\
\hline \multicolumn{7}{|c|}{ P. aeruginosa ATCC 10145} \\
\hline Morphotype I & TSA & Circular & Irregular & Rough and wrinkled & Large & Yellow \\
\hline Morphotype II & PIA & Circular & Irregular & Wrinkled and rough & Large & Green \\
\hline Morphotype III & CET & Circular & Irregular & Rough & Large & Green \\
\hline \multicolumn{7}{|l|}{ Isolated $P$. aeruginosa } \\
\hline Morphotype IV & TSA & Circular & Entire & Smooth & Large & Yellow \\
\hline Morphotype V & TSA & Circular & Irregular & Smooth & Large & Yellow \\
\hline Morphotype III & PIA & Circular & Irregular & Rough & Large & Green \\
\hline Morphotype VI & CET & Irregular & Irregular & Rough and smooth & Large & Green \\
\hline \multicolumn{7}{|l|}{ Biofilm } \\
\hline \multicolumn{7}{|c|}{ P. aeruginosa ATCC 10145} \\
\hline Morphotype I & TSA & Circular & Irregular & Rough and wrinkled & Large & Yellow \\
\hline Morphotype VII & TSA & Circular & Entire & Wrinkled & Large & Yellow \\
\hline Morphotype VIII & TSA & Circular & Irregular & Rough & Large & Yellow \\
\hline Morphotype II & PIA & Circular & Irregular & Wrinkled and rough & Large & Green \\
\hline Morphotype IX & PIA & Circular & Entire & Wrinkled and rough & Small & Green \\
\hline Morphotype III & CET & Circular & Irregular & Rough & Large & Green \\
\hline Morphotype X & CET & Circular & Entire & Rough & Small & Green \\
\hline \multicolumn{7}{|l|}{ Isolated $P$. aeruginosa } \\
\hline Morphotype IV & TSA & Circular & Entire & Smooth & Large & Yellow \\
\hline Morphotype V & TSA & Circular & Irregular & Smooth & Large & Yellow \\
\hline Morphotype XI & TSA & Irregular & Irregular & Wrinkled & Small & Yellow \\
\hline Morphotype XII & TSA & Circular & Entire & Rough and wrinkled & Small & Yellow \\
\hline Morphotype XIII & TSA & Circular & Entire & Smooth and wrinkled & Small & Yellow \\
\hline Morphotype XIV & TSA & Circular & Irregular & Smooth and wrinkled & Small & Yellow \\
\hline Morphotype XV & TSA & Circular & Irregular & Wrinkled & Small & Yellow \\
\hline Morphotype XVI & TSA & Circular & Irregular & Rough and wrinkled & Small & Yellow \\
\hline Morphotype XVII & TSA & Circular & Entire & Smooth & Small & Yellow \\
\hline Morphotype XVIII & TSA & Circular & Entire and irregular & Smooth and wrinkled & Small & Yellow \\
\hline Morphotype III & PIA & Circular & Irregular & Rough & Large & Green \\
\hline Morphotype IX & PIA & Circular & Entire & Wrinkled and rough & Small & Green \\
\hline Morphotype VI & CET & Irregular & Irregular & Rough and smooth & Large & Green \\
\hline Morphotype X & CET & Circular & Entire & Rough & Small & Green \\
\hline
\end{tabular}

a Texture should be described from out to inside; morphotypes with same types of texture but in different zones were considered as distinct colony variants.

b Colonies were considered small if presented diameter is below $3 \mathrm{~mm}$ and large if presented diameter is above $3 \mathrm{~mm}$.

faecalis (Qamer et al., 2003; Wellinghausen et al., 2009). The gain of knowledge about phenomena involved in bacterial adaptation and survival such as phenotypic switching, biofilm resistance, bacterial persistence and other biological processes may be compromised since the impact of experimental conditions in colony morphology definition is still unknown. The present study shows the impact of experimental factors, including colony growth time, plate colony density, culture medium, planktonic or sessile mode of growth and genetic background, on morphological features of $P$. aeruginosa colonies. The monitoring of the colony development over time demonstrated that, in the case of $P$. aeruginosa colonies, morphological characterization should just be performed after, at least, $45 \mathrm{~h}$ of colonial growth. Characteristic morphological features remain unchanged only after $45 \mathrm{~h}$ of growth (Figs. 1, 2, 3 and 4), and by this reason the assessment of $P$. aeruginosa colonies with less than $45 \mathrm{~h}$ of growth led to inaccurate characterizations and further misinterpretations. Several studies addressed observations of $P$. aeruginosa colonies with less than $45 \mathrm{~h}$, which may raise the question whether the authors would achieve the same conclusions observing older colonies (Hay et al., 2009; Kirisits et al., 2005).

The diversity of SCV observed and described in the present study (Fig. 4A-H) supports as well the need of colonial characterization just when colonies reached the definitive and unchanged state. In literature, no studies reported high SCV heterogeneity besides the rough SCV possibly due to limited colony growth time allowed (Deziel et al., 2001; Drenkard and Ausubel, 2002; Kirisits et al., 2005). The series of SCV presented in this study may play a relevant role on biofilm resistance or persistence to environmental stressors unknown until now, possibly, due to the improper colony characterization. Based on the data disclosed in the present study and to prevent the possible loss of data about colony morphology, a previous study of colony development before the main biological study, is recommended. This would allow determining the duration of complete colony morphogenesis and no morphotypes will be undetected and mischaracterized.

Several reports exhibit images of colonies, many of them with various colonies surrounding each other (Boles et al., 2004; Haussler et al., 2003; Smania et al., 2004). The present data showed that distance between colonies, an issue frequently ignored, is important. Neighbouring colonies might limit or alter morphogenesis possibly due to competition for nutritional resources or bacterial signalling and communication. Be'er et al. (2009) reported that sibling colonies decelerate or even stop their growth when facing each other and stated that the production and perception of small signalling molecules may influence colony morphogenesis, gene expression and cell differentiation. Therefore, the characterization of colonies properly separated is recommended.

The solid media used to develop bacterial colonies is also a condition of great discrepancy among reports. Regarding P. aeruginosa colonies, media such as TSA, LB agar, PIA and blood agar have been used to observe colony morphology variation (Flemming et al., 2007; Rakhimova et al., 2008; Starkey et al., 2009). It is well known that solid media composition influences fungal colony morphogenesis (Fries et al., 2002). Concerning bacterial colonies, some reports had demonstrated colony morphology dependency on nutritional and agar concentration in the scope of colony pattern modelling (Bonachela et al., 2011; Matsushita et al., 1999; Matsuyama and Matsushita, 2001). However, the real impact of these variations on each of the morphological characteristics, including size, form, colour, texture and margin, has not been explored. The preservation of morphological features is critical, for instance, in clinical diagnosis. Typically, clinical samples from patients are plated on solid media and the detection of certain morphotypes such as SCV is crucial to design an effective therapy. Thus, the question of whether 
Table 3

Guidelines to accurately perform and compare results among bacterial colony morphology observations.

\begin{tabular}{|c|c|}
\hline Solid media & $\begin{array}{l}\text { The medium composition used to plate bacteria should be taken into account: comparisons should only be performed when the solid medium } \\
\text { is identical. }\end{array}$ \\
\hline Colony growth time & $\begin{array}{l}\text { A prior study should be performed before colony morphology characterization; colony growth time is established for the time from which all } \\
\text { morphological traits are constant over the time, except the size. }\end{array}$ \\
\hline Colony density per plate & $\begin{array}{l}\text { Colony morphology observation should not be performed using plates with increased number of colonies; there is not a general threshold because } \\
\text { colony size is highly dependent on the bacterial species. For instance, P. aeruginosa colonies are typically large and observation should be performed } \\
\text { in plate with less than } 15 \text { colonies. }\end{array}$ \\
\hline Bacterial strain & $\begin{array}{l}\text { Some reports and manuals indicate typical morphological patterns for bacterial species, however different genetic background and biological } \\
\text { phenomena as phenotypic switching may alter the typical patterns of a bacterial species. Therefore, previous colony morphogenesis study } \\
\text { should be always performed. }\end{array}$ \\
\hline Number of replicates & $\begin{array}{l}\text { As other microbiological studies, colony morphology characterization should be performed at least } 3 \text { times. However, } 5 \text { independent assays are } \\
\text { recommended to avoid isolate random variants that may arise. }\end{array}$ \\
\hline
\end{tabular}

the detection of SCV is affected by the solid media used, i.e., dependent of nutritional composition of media, is of critical importance. Results obtained showed that the composition of the solid media used to plate $P$. aeruginosa, either coming from planktonic or biofilm cultures, is relevant to colony morphology definition. The effect of medium composition in colony patterns was clearly evident in traits as margin, colour and texture (Table 2). In addition, the present results evidenced that bacteria spread onto non-selective medium as TSA generate more colony morphology diversity, in contrast with Pseudomonas selective agar such as PIA and CET. For instance, the expression of SCV morphotype from clinical isolated biofilm-cells on TSA was higher, eight SCV were detected, in contrast with just one morphotype on PIA and CET. This is maybe explained by the presence of irgasan in PIA and nalidixic acid and cetrimide in CET that may inhibit the growth of some colony variants (Fonseca et al., 1986). The perception of colony morphologies dependent of nutrient concentration challenges the traditional morphology-based methods to identify bacteria and may affect the actual performance of clinical diagnosis culture dependent approaches.

Considering all the issues previously discussed, a set of guidelines is proposed for authors, clinicians and technicians to implement when performing colony morphology characterization and further comparing results (Table 3). Scientific research and clinical diagnosis are the most benefited with similarity of the experimental procedures of the colony morphology method. This will lead to better comprehension of bacterial adaptation and evolution, purposes of fundamental science, with the ultimate goal of predicting antimicrobial resistance, expression of virulence factors and persistence ability based on morphological traits, relevant for supporting clinical diagnosis on bacterial profiling.

\section{References}

Agarwal, G., Kapil, A., Kabra, S.K., Das, B.K., Dwivedi, S.N., 2005. Characterization of Pseudomonas aeruginosa isolated from chronically infected children with cystic fibrosis in India. BMC Microbiol. 5, 43.

Allegrucci, M., Sauer, K., 2007. Characterization of colony morphology variants isolated from Streptococcus pneumoniae biofilms. J. Bacteriol. 189, 2030-2038.

Balaban, N.Q., Merrin, J., Chait, R., Kowalik, L., Leibler, S., 2004. Bacterial persistence as a phenotypic switch. Science 305, 1622-1625.

Be'er, A., Florin, E.L., Fisher, C.R., Swinney, H.L., Payne, S.M., 2011. Surviving bacterial sibling rivalry: inducible and reversible phenotypic switching in Paenibacillus dendritiformis. mBio 2, e00069-e.

Be'er, A., et al., 2009. Deadly competition between sibling bacterial colonies. Proc. Natl. Acad. Sci. U. S. A. 106 (2), 428-433.

Boles, B.R., Thoendel, M., Singh, P.K., 2004. Self-generated diversity produces 'insurance effects' in biofilm communities. Proc. Natl. Acad. Sci. U. S. A. 101, 16630-16635.

Bonachela, J.A., Nadell, C.D., Xavier, J.B., Levin, S.A., 2011. Universality in bacterial colonies. J. Stat. Phys. 144, 303-315.

Braga, P.A.C., Tata, A., Goncalves dos Santos, V., Barreiro, J.R., Schwab, N.V., Veiga dos Santos, M., Eberlin, M.N., Ferreira, C.R., 2013. Bacterial identification: from the agar plate to the mass spectrometer. RSC Adv. 3, 994-1008.

Chantratita, N., Wuthiekanun, V., Boonbumrung, K., Tiyawisutsri, R., Vesaratchavest, M. Limmathurotsakul, D., Chierakul, W., Wongratanacheewin, S., Pukritiyakamee, S., White, N.J., Day, N.P., Peacock, S.J., 2007. Biological relevance of colony morphology and phenotypic switching by Burkholderia pseudomallei. J. Bacteriol. 189, 807-817.

Costerton, J.W., Cheng, K.J., Geesey, G.G., Ladd, T.I., Nickel, J.C., Dasgupta, M., Marrie, T.J., 1987. Bacterial biofilms in nature and disease. Annu. Rev. Microbiol. 41, 435-464.

Davies, D., 2003. Understanding biofilm resistance to antibacterial agents. Nat. Rev. Drug Discov. 2, 114-122.
Davies, J.A., Harrison, J.J., Marques, L.L., Foglia, G.R., Stremick, C.A., Storey, D.G., Turner, R.J., Olson, M.E., Ceri, H., 2007. The GacS sensor kinase controls phenotypic reversion of small colony variants isolated from biofilms of Pseudomonas aeruginosa PA14. FEMS Microbiol. Ecol. 59, 32-46.

Deziel, E., Comeau, Y., Villemur, R., 2001. Initiation of biofilm formation by Pseudomonas aeruginosa 57RP correlates with emergence of hyperpiliated and highly adherent phenotypic variants deficient in swimming, swarming, and twitching motilities 1. J. Bacteriol. 183, 1195-1204.

Donlan, R.M., 2002. Biofilms: microbial life on surfaces. Emerg. Infect. Dis. 8, 881-890.

Drenkard, E., 2003. Antimicrobial resistance of Pseudomonas aeruginosa biofilms. Microbes Infect. 5, 1213-1219.

Drenkard, E., Ausubel, F.M., 2002. Pseudomonas biofilm formation and antibiotic resistance are linked to phenotypic variation. Nature 416, 740-743.

Flemming, H.C., Neu, T.R., Wozniak, D.J., 2007. The EPS matrix: the "house of biofilm cells". J. Bacteriol. 189, 7945-7947.

Fonseca, K., MacDougall, J., Pitt, T.L., 1986. Inhibition of Pseudomonas aeruginosa from cystic fibrosis by selective media. J. Clin. Pathol. 39, 220-222.

Fries, B.C., Goldman, D.L., Casadevall, A., 2002. Phenotypic switching in Cryptococcus neoformans. Microbes Infect. 4, 1345-1352.

Goerke, C., Gressinger, M., Endler, K., Breitkopf, C., Wardecki, K., Stern, M., Wolz, C., Kahl, B.C., 2007. High phenotypic diversity in infecting but not in colonizing Staphylococcus aureus populations. Environ. Microbiol. 9, 3134-3142.

Haussler, S., 2004. Biofilm formation by the small colony variant phenotype of Pseudomonas aeruginosa. Environ. Microbiol. 6, 546-551.

Haussler, S., Tummler, B., Weissbrodt, H., Rohde, M., Steinmetz, I., 1999. Small-colony variants of Pseudomonas aeruginosa in cystic fibrosis. Clin. Infect. Dis. 29, 621-625.

Haussler, S., Ziegler, I., Lottel, A., von Gotz, F., Rohde, M., Wehmhohner, D., Saravanamuthu, S., Tummler, B., Steinmetz, I., 2003. Highly adherent small-colony variants of Pseudomonas aeruginosa in cystic fibrosis lung infection. J. Med. Microbiol. $52,295-301$.

Hay, I.D., Remminghorst, U., Rehm, B.H., 2009. MucR, a novel membrane-associated regulator of alginate biosynthesis in Pseudomonas aeruginosa. Appl. Environ. Microbiol. 75, 1110-1120.

Hilmi, D., Parcina, M., Bode, K., Ostrop, J., Schuett, S., Heeg, K., Ziebuhr, W., Sommerburg, O., Bekeredjian-Ding, I., 2013. Functional variation reflects intra-strain diversity of Staphylococcus aureus small colony variants in the host-pathogen interaction. Int. J. Med. Microbiol. 303, 61-69.

Hoffman, L.R., Deziel, E., D'Argenio, D.A., Lepine, F., Emerson, J., McNamara, S., Gibson, R.L., Ramsey, B.W., Miller, S.I., 2006. Selection for Staphylococcus aureus small-colony variants due to growth in the presence of Pseudomonas aeruginosa. Proc. Natl. Acad. Sci. U. S. A. 103, 19890-19895.

Hogardt, M., Heesemann, J., 2010. Adaptation of Pseudomonas aeruginosa during persistence in the cystic fibrosis lung. Int. J. Med. Microbiol. 300, 557-562.

Kirisits, M.J., Prost, L., Starkey, M., Parsek, M.R., 2005. Characterization of colony morphology variants isolated from Pseudomonas aeruginosa biofilms. Appl. Environ. Microbiol. 71, 4809-4821.

Lewis, K., 2001. Riddle of biofilm resistance. Antimicrob. Agents Chemother. 45, 999-1007.

Lewis, K., 2005. Persister cells and the riddle of biofilm survival. Biochemistry (Mosc) 70, 267-274.

Lyczak, J.B., Cannon, C.L., Pier, G.B., 2002. Lung infections associated with cystic fibrosis. Clin. Microbiol. Rev. 15, 194-222.

Manno, G., Cruciani, M., Romano, L., Scapolan, S., Mentasti, M., Lorini, R., Minicucci, L., 2005. Antimicrobial use and Pseudomonas aeruginosa susceptibility profile in a cystic fibrosis centre. Int. J. Antimicrob. Agents 25, 193-197.

Martin, D.W., Schurr, M.J., Mudd, M.H., Govan, J.R., Holloway, B.W., Deretic, V., 1993. Mechanism of conversion to mucoidy in Pseudomonas aeruginosa infecting cystic fibrosis patients. Proc. Natl. Acad. Sci. U. S. A. 90, 8377-8381.

Massey, R.C., Buckling, A., Peacock, S.J., 2001. Phenotypic switching of antibiotic resistance circumvents permanent costs in Staphylococcus aureus. Curr. Biol. 11, 1810-1814.

Matsushita, M., Wakita, J., Itoh, H., Watanabe, K., Arai, T., Matsuyama, T., Sakaguchi, H. Mimura, M., 1999. Formation of colony patterns by a bacterial cell population. Physica A 274, 190-199.

Matsuyama, T., Matsushita, M., 2001. Population morphogenesis by cooperative bacteria. Forma 16, 307-326.

Norstrom, T., Lannergard, J., Hughes, D., 2007. Genetic and phenotypic identification of fusidic acid-resistant mutants with the small-colony-variant phenotype in Staphylococcus aureus. Antimicrob. Agents Chemother. 51, 4438-4446. 
Proctor, R.A., von Eiff, C., Kahl, B.C., Becker, K., McNamara, P., Herrmann, M., Peters, G. 2006. Small colony variants: a pathogenic form of bacteria that facilitates persistent and recurrent infections. Nat. Rev. Microbiol. 4, 295-305.

Qamer, S., Sandoe, J.A., Kerr, K.G., 2003. Use of colony morphology to distinguish different enterococcal strains and species in mixed culture from clinical specimens. J. Clin. Microbiol. 41, 2644-2646.

Rakhimova, E., Munder, A., Wiehlmann, L., Bredenbruch, F., Tummler, B., 2008. Fitness of isogenic colony morphology variants of Pseudomonas aeruginosa in murine airway infection. PLoS ONE 3, e1685

Rossignol, G., Sperandio, D., Guerillon, J., Duclairoir Poc, C., Soum-Soutera, E., Orange, N., Feuilloley, M.G., Merieau, A., 2009. Phenotypic variation in the Pseudomonas fluorescens clinical strain MFN1032. Res. Microbiol. 160, 337-344.

Sauer, K., Camper, A.K., Ehrlich, G.D., Costerton, J.W., Davies, D.G., 2002. Pseudomona aeruginosa displays multiple phenotypes during development as a biofilm. J. Bacteriol. $184,1140-1154$.

Schneider, M., Muhlemann, K., Droz, S., Couzinet, S., Casaulta, C., Zimmerli, S., 2008. Clinical characteristics associated with isolation of small-colony variants of Staphylococcus aureus and Pseudomonas aeruginosa from respiratory secretions of patients with cystic fibrosis. J. Clin. Microbiol. 46, 1832-1834.

Singh, R., Ray, P., Das, A., Sharma, M., 2009. Role of persisters and small-colony variants in antibiotic resistance of planktonic and biofilm-associated Staphylococcus aureus: an in vitro study. J. Med. Microbiol. 58, 1067-1073.

Smania, A.M., Segura, I., Pezza, R.J., Becerra, C., Albesa, I., Argarana, C.E., 2004. Emergence of phenotypic variants upon mismatch repair disruption in Pseudomonas aeruginosa Microbiology 150, 1327-1338.

Sousa, A.M., Machado, I., Pereira, M.O., 2011. Phenotypic switching: an opportunity to bacteria thrive. In: Mendez-Vilas, A. (Ed.), Science Against Microbial Pathogens: Communicating Current Research and Technological Advances.
Spoering, A.L., Lewis, K., 2001. Biofilms and planktonic cells of Pseudomonas aeruginosa have similar resistance to killing by antimicrobials 1 . J. Bacteriol. 183, 6746-6751.

Starkey, M., Hickman, J.H., Ma, L., Zhang, N., De Long, S., Hinz, A., Palacios, S., Manoil, C., Kirisits, M.J., Starner, T.D., Wozniak, D.J., Harwood, C.S., Parsek, M.R., 2009. Pseudomonas aeruginosa rugose small-colony variants have adaptations that likely promote persistence in the cystic fibrosis lung. J. Bacteriol. 191, 3492-3503.

Stepanovic, S., Vukovic, D., Dakic, I., Savic, B., Svabic-Vlahovic, M., 2000. A modified microtiter-plate test for quantification of staphylococcal biofilm formation. J. Microbiol. Meth. 40, 175-179.

Stewart, P.S., Franklin, M.J., 2008. Physiological heterogeneity in biofilms. Nat. Rev. Microbiol. 6 (3), 199-210.

Stewart, P.S., 2002. Mechanisms of antibiotic resistance in bacterial biofilms. Int. J. Med. Microbiol. 292, 107-113.

Tannaes, T., Grav, H.J., Bukholm, G., 2000. Lipid profiles of Helicobacter pylori colony variants. APMIS 108, 349-356.

Weile, J., Knabbe, C., 2009. Current applications and future trends of molecular diagnostics in clinical bacteriology. Anal. Bioanal. Chem. 394, 731-742.

Weiser, J.N., Markiewicz, Z., Tuomanen, E.I., Wani, J.H., 1996. Relationship between phase variation in colony morphology, intrastrain variation in cell wall physiology, and nasopharyngeal colonization by Streptococcus pneumoniae. Infect. Immun. 64, 2240-2245.

Wellinghausen, N., Chatterjee, I., Berger, A., Niederfuehr, A., Proctor, R.A., Kahl, B.C., 2009. Characterization of clinical Enterococcus faecalis small-colony variants. J. Clin. Microbiol. 47, 2802-2811.

Yachi, S., Loreau, M., 1999. Biodiversity and ecosystem productivity in a fluctuating environment: the insurance hypothesis. Proc. Natl. Acad. Sci. U. S. A. 96, 1463-1468. 